\title{
Automatic network-level bridge monitoring by integration of InSAR and GIS catalogues
}

\author{
Luca Bianchini Ciampoli*a, Valerio Gagliardi ${ }^{\mathrm{a}}$, Alessandro Calvi ${ }^{\mathrm{a}}$, \\ Fabrizio D'Amico ${ }^{\mathrm{a}}$ and Fabio Tosti ${ }^{\mathrm{b}}$ \\ a'Dipartimento di Ingegneria, Roma Tre University, Via Vito Volterra 62, 00146, Rome, Italy; \\ ${ }^{\mathrm{b}} \mathrm{School}$ of Computing and Engineering, University of West London \\ St Mary's Road, W55RF London, United Kingdom
}

\begin{abstract}
Smart monitoring of critical civil engineering infrastructures has become a priority nowadays as ageing of construction materials may have dramatic consequences on the community. The issue is exacerbated as it applies to many structures at the network level rather than to single structures or limited areas. To this effect, catalogues for assessment of decay conditions and identification of maintenance actions are crucial pieces of information for infrastructure management purposes.

Within this context, innovative non-destructive methods, such as space-borne techniques, have been increasingly used for monitoring purposes in the past two decades. Among these, the Interferometric Synthetic Aperture Radar (InSAR) imagery technique is gaining momentum nowadays. This method is used to monitor ground and infrastructure displacements at the large scale with a millimeter resolution. It can compare radar satellite images over time, and it is capable to measure variations accurately using interferometry. An advantage of using InSAR techniques is that these are not affected by cloudiness as well as lighting conditions, since data can be also collected at night time. On the contrary, InSAR is computational-demanding as it requires to filter out unnecessary information from the entire captured area to obtain data on the target domain. Most common applications include landslide assessment and monitoring of surface deformations following major seismic events.

This paper reports a methodology for the assessment of surface deformations of viaducts by reducing drastically computational time. To this purpose, a multi-stage automatic bridge monitoring protocol is developed at the network level by integration of information into Geographic Information System (GIS) catalogues and use of the InSAR imagery technique. The first stage locates the viaducts in the area of interest by querying open data and inputting results into a GIS catalogue. On a second stage, an InSAR analysis of the identified bridges is performed. This approach allows an estimate of surface displacements as well as an identification of bridge areas affected by millimeter-scale settlements.

Fundamental theoretical and working principles of the two methodologies are first introduced in the paper. Advantages against drawbacks of each technique are then discussed. The last Section reports a case study and a discussion of the main results including conclusions and future prospects.
\end{abstract}

Keywords: network-level bridge monitoring; InSAR; GIS; multi-stage integrated methodology; critical infrastructure maintenance

\section{INTRODUCTION}

Recent and dramatic events occurred on the European transport network, e.g. viaduct collapses or convoy derailments, have pointed out the urgent need for assessing the actual state of health along transport assets. Similar considerations can be formulated regarding the high level of exposition and vulnerability of transport systems towards natural hazards. To this effect, bridges and viaducts are worthy of mention. Failure or collapse of bridges involve severe risks for human safety, and might segregate entire geographical areas from any transport connection. To provide proper connection of the transport network, mostly in territories with an unfavorable geomorphology, design service life of bridges is generally more extended, typically 50 years. Health monitoring of bridges and viaducts is a crucial activity for preventing unexpected structural collapses. In fact, it is evident that a proper management is crucial to prevent structural failures and ensure serviceability of the asset. 
It is worthy of mention that out of 600 '000 bridges subject to inspection in 2002 in the US, $40 \%$ were reported to be structural deficient or not working [1]. Structural health monitoring activities for bridges generally aim at [2]:

1. validating design assumptions and parameters with the aim of improving design guidelines for future constructions;

2. detecting anomalies in the mechanical response of the structure, involving early-stage decays that might compromise the structural stability;

3. providing real-time or quasi-real-time information for assessing safety conditions immediately after an hazardous event;

4. providing useful information to prioritize rehabilitation works;

5. assessing the viability of maintenance activities through on-site monitoring;

6. collecting comprehensive datasets for field-related activities.

The development of a comprehensive monitoring system capable to achieve all the above tasks is still a challenge to date. In this regard, different types of non-destructive testing (NDT) techniques are commercially available [3]. To cite a few, accelerometers, smart total station, vibration measurement sensors, wireless network systems, ground-penetrating radar and space-borne and ground-based interferometers have proven to be effective for purpose.

Within this context, this study reports an innovative procedure that allows an integrated use of different non-destructive monitoring techniques, to facilitate an effective management of linear transport infrastructures. To this purpose, a multistage automatic bridge monitoring protocol was developed at the network level by integration of information into Geographic Information System (GIS) catalogues with Interferometric Synthetic Aperture Radar (InSAR) imagery.

The first stage of the method locates viaducts in the area of interest by querying open data and inputting results into a GIS catalogue. On a second stage, an InSAR analysis of identified bridges is performed. This approach allows an estimate of surface displacements and an identification of bridge areas affected by millimeter-scale settlements. It allows to minimise computational efforts required to process the data.

\section{METHODOLOGY}

\subsection{The Geographic Dataset}

It is known that technical registers with full details of bridges and viaducts of a transport network are rarely available to administrations. To this effect, there is a need to create an informative catalogue of structures with the aim to collect information on their actual number and geographic location.

In this study, the above aim was achieved by using the following tools:

- OpenStreetMap (OSM) [4]: an open data geographic database gathering information acquired from GPS devices, aerial photos and other free-source tools that are voluntarily uploaded by users;

- OverpassTurbo [5]: a web-based application that allows to run queries based on Application Programming Interface (API). This allows to search for specific elements in OSM over an inspected area, and to export the georefernced outputs;

- QGIS [6]: an open-source software capable to manage several geographic input data for territorial analysis purposes.

It is possible to run specific searches for bridges and viaducts within an identified area by giving the corresponding script as an input to the Overpass Turbo application. Results from the search for bridges over the area of the Lazio Region, in central Italy, are reported in Fig. 1 along with the relevant script. 


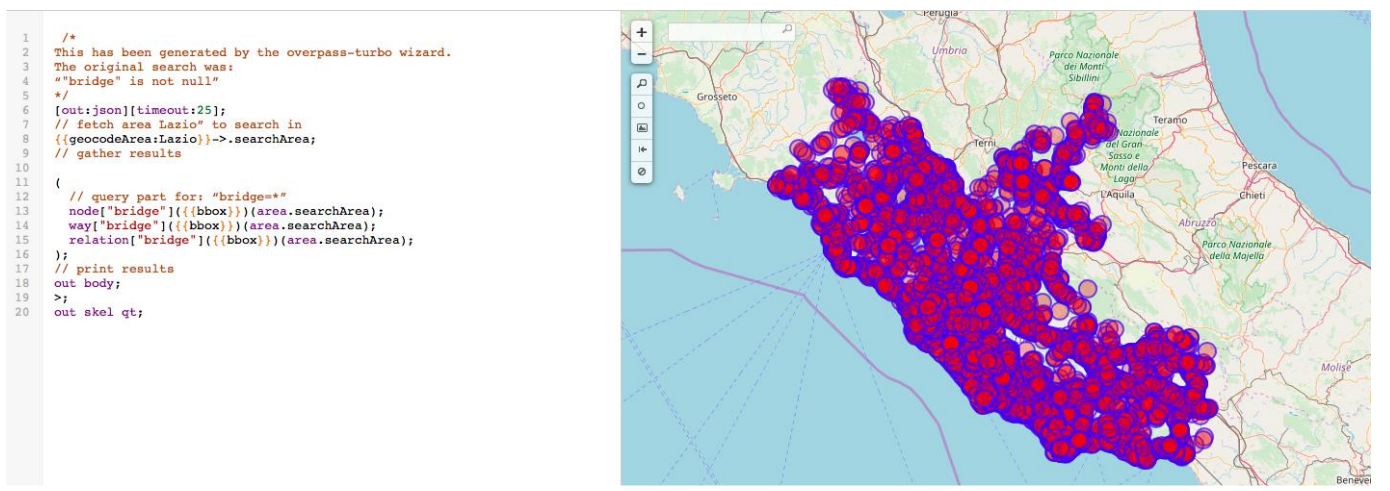

Figure 1. Search for bridges over the area of Lazio Region, central Italy, in Overpass Turbo.

The dataset shown in Fig. 1 is in a raw format. To fully exploit its informative content, a data processing stage is required. Potential multiple values referred to a same viaduct, typically occurring in the case of a multi-carriageway bridge, are among the potential issues that need to be taken into account. To overcome this occurrence, the following conditions were constrained for each structure:

$$
\begin{aligned}
& L_{b, i}-L_{b, j}<3 m \\
& \text { Lat }_{b, i}-\text { Lat }_{b, j}<0.003^{\circ} \\
& \text { Long }_{b, i}-\text { Long }_{b, j}<0.003^{\circ}
\end{aligned}
$$

Where L, Lat and Long are the length, the latitude and the longitude of the $i^{\text {th }}$ and the $j^{\text {th }}$ structure, respectively. Once unnecessary records are filtered out from the dataset, it is possible to import the ensemble of bridges and viaducts into the Q-GIS software. As an example, the effect of the application of the above filter to a railway bridge dataset is shown in Fig. 2.

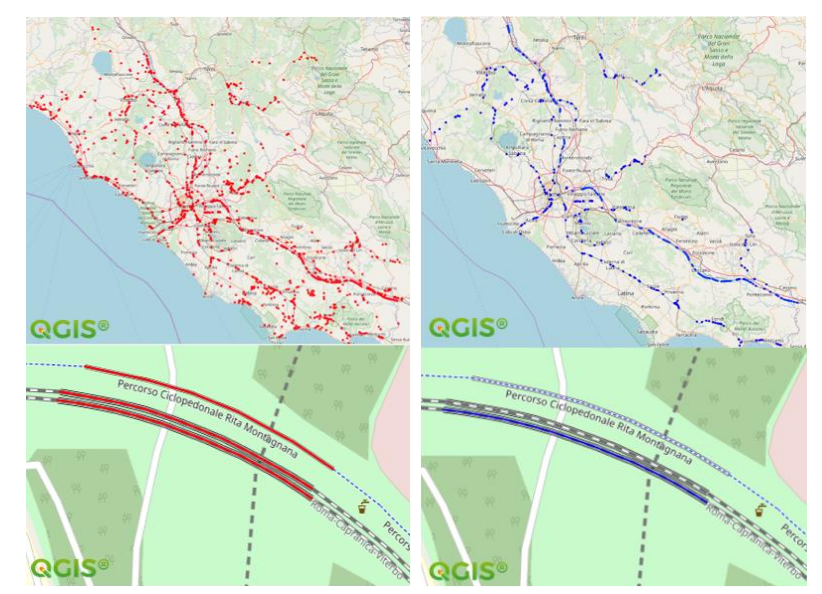

Figure 2. Visualisation of railway bridges and viaducts in the Lazio Region, central Italy, in QGIS before (red) and after (blue) the data filtering.

\subsection{The InSAR Technique}

Synthetic Aperture Radar (SAR) can obtain electromagnetic images of the Earth's surface. These systems are active satellite devices that emit radar pulses with frequencies ranging between $0.23 \mathrm{GHz}$ and $40 \mathrm{GHz}$ and receive the backscattered field produced by the interactions of the EM waves and the ground surface.

Differently from usual radars, the SAR technique uses orbiting satellites to observe same territory areas from progressive positions. In this manner, they can synthetise larger antennas and increase the resolution on the ground. 
SAR sensors operate at different bands of the EM spectrum, namely X, C, and L bands, corresponding to wavelengths ( $\lambda$ ) ranging from $2.4 \mathrm{~cm}$ to $30 \mathrm{~cm}$. The different wavelengths affect the interactions between the EM field and the elements on the surface and, therefore, the backscatter of the signal.

Interferometric Synthetic Aperture Radar (InSAR) techniques, or SAR Interferometry, consist of measuring the variation of the EM signal phase between images acquired at different time over a same area. Indeed, as a vertical displacement of the ground occurs, the distance between the sensor and the ground varies accordingly, thereby affecting the phase of the signal back-received by the sensor.

In more detail, an interferogram is generated by calculating the phase difference between two SAR products of the same land portion. This corresponds to a matrix of numerical values ranging from $-\pi$ to $+\pi$. Thus, the evaluation of an interferogram allows to identify potential displacements over an investigated area during the observation time, by observing the variation within a numerical matrix.

However, the analysis of a SAR interferogram is a complex operation, since the interferometric phase is composed of various different signal contributions, including noise and decorrelation effects [7].

Specifically, an interferometric phase $(\Delta \varphi)$ is affected by four main contributions, expressed in the equation below:

$$
\Delta \varphi=4 \pi / \lambda \Delta R+\alpha+t+\text { noise }
$$

with $\lambda$ being the wavelength of the emitted signal, $\Delta R$ the displacement in the Line Of Sight (LOS) and where $\alpha$ is a phase shift due to different atmospheric conditions (e.g. moisture in suspension); $t$ accounts for the local topography and the noise term includes the contributions of the temporal change of the scatterers and different looking angles. Specifically, the first term is referred to as "propagator" [7,8] and it directly depends on sensor-target distance variation.

In general, the target of the technique is to identify all the single phase terms to isolate the phase contributions that are not related to signal disturbances and, accordingly, can be related to ground displacements only.

Among the various InSAR techniques, the Permanent Scatterer Interferometry (PSI) method is based on the selection of a small subset of radar targets, named permanent scatterers (PSs) that are found to show no decorrelation phenomena, over the observation time. These targets maintain phase coherence over successive SAR acquisitions, and can be used to effectively reconstruct and compensate the atmospheric disturbance on the entire radar image, as weather conditions slowly vary in space, and are not correlated over time.

A dataset of at least 20 SAR images of an area showing a sufficient spatial density of PS [9] is necessary to successfully perform a PS analysis. In practical terms, the technique works by applying the steps reported in Fig. 3.

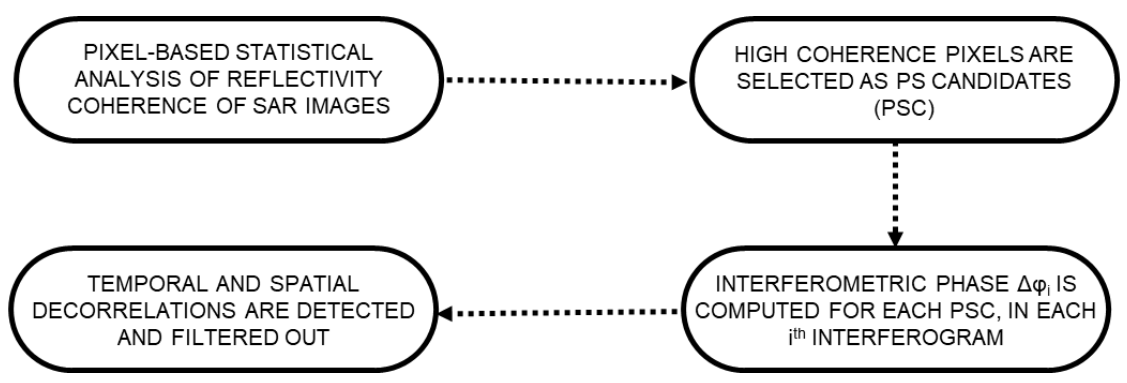

Figure 3. Typical steps performed in a PS analysis.

As a result, stable reflectors, i.e. PS, can be detected over the inspected area, allowing surface displacement velocities to be measured with millimeter accuracy $[9,10]$. At the end of the process, displacement evolution trends can be generated for every PS, and an average velocity map can be produced to provide an overview of the average ground motion over an area of interest. 


\subsection{Data Integration}

The integration of the two information allows to conduct an automatic displacement analysis on the overall amount of bridges and viaducts in a given network, at the required scale. A scheme of the workflow is synthetically shown in Fig. 3.

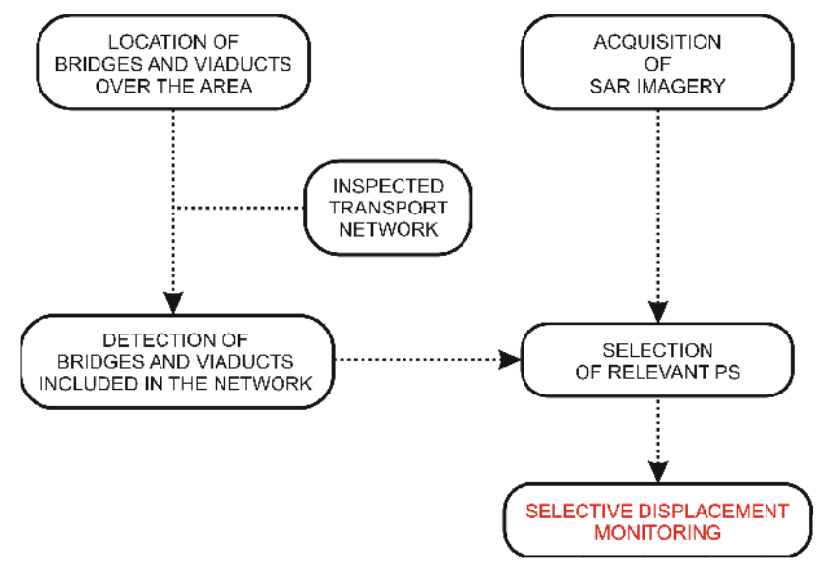

Figure 4. Scheme of the adopted workflow.

Specifically, the overall number of bridges and viaducts is detected by querying open data and inputting results into a GIS. Once the dataset is preliminarily filtered of double values and invalid data, the coordinates of the single items are compared to the transport network taken into account (e.g., railways, highways, freeways). As a result, bridges and viaducts related to the specific network are only selected out of the overall ensemble.

In parallel, SAR imagery is acquired and processes in order to product the PS over the entire area. By combining this dataset with the position of bridges within the inspected network, it is then possible to select the only PS useful for monitoring displacement of a single structure.

Matching between SAR and GIS database were performed in the MATLAB environment, and complied with the following condition:

$$
D_{i, j}<30 m
$$

Where $D$ is the Haversine distance between the $i^{\text {th }}$ PS and the $j^{\text {th }}$ bridge, expressed as follows:

$$
D_{i, j}=2 R \sin ^{-1}\left(\sqrt{\sin ^{2}\left(\frac{\text { Lat }_{j}-\text { Lat }_{i}}{2}\right)+\cos \left(\text { Lat }_{i}\right) \cos \left(\text { Lat }_{j}\right) \sin ^{2}\left(\frac{\text { Long }_{j}-\text { Long }_{i}}{2}\right)}\right)
$$

with $R$ being the average radius of the Earth. As a consequence, all the PS more distant than $30 \mathrm{~m}$ from a bridge or viaduct are discharged by the procedure.

\section{EXPERIMENTAL FRAMEWORK}

The aforementioned methodology was tested over an actual transport network. Specifically, both geographical detection of bridges and viaducts and PS analyses were conducted over the area of Lazio Region, in central Italy, with particular reference to the railway bridges and viaducts.

Out of 1624 records obtained by querying the geographic catalogues, 509 items were actually identified as bridges or viaducts after the filtering of data. Fig. 4 shows the location of the structures over the inspected territory (in red), with respect to the actual railway network (in black). 


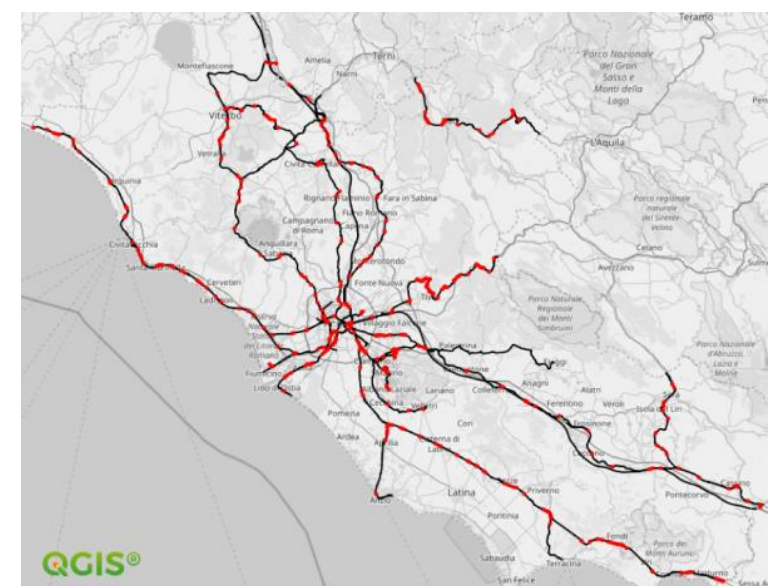

Figure 5. Detection of railway bridges and viaducts over the area of Lazio Region, central Italy.

In this study, a PS dataset provided by the Italian Ministry for Environment and Territory and Sea Safeguard (MATTM) in the framework of the Extraordinary Environmental Remote Sensing Plan (PST-A) [11] was employed.

Specifically, a total of 45 Stripmap images of the $1700 \mathrm{~km} 2$-wide territory were collected, for a survey period ranging between April 2011 and March 2014. The data had been collected in ascending and descending geometries from COSMOSkyMed mission, having a spatial resolution of $3 \mathrm{~m}$ x $3 \mathrm{~m}$. Example of PS analysis outcome is reported in Fig. 5, at different scale, with reference to a single satellite sweep.
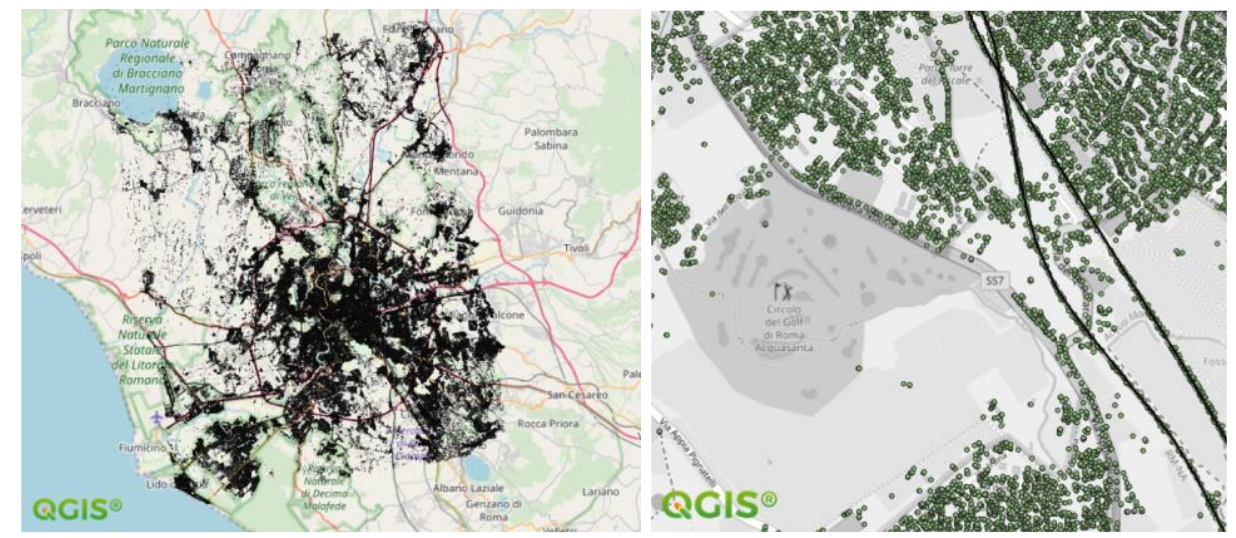

Figure 6. Outcomes of PS analysis.

\section{RESULTS}

The different datasets shown in Figures 4 and 5 were finally integrated for achieving aThe application of the presented methodology effectively allowed reducing the number of PS to analyze to only those actually pertaining to the inspected railway network. Examples of the positive outcome of the application are reported in Fig. 6.
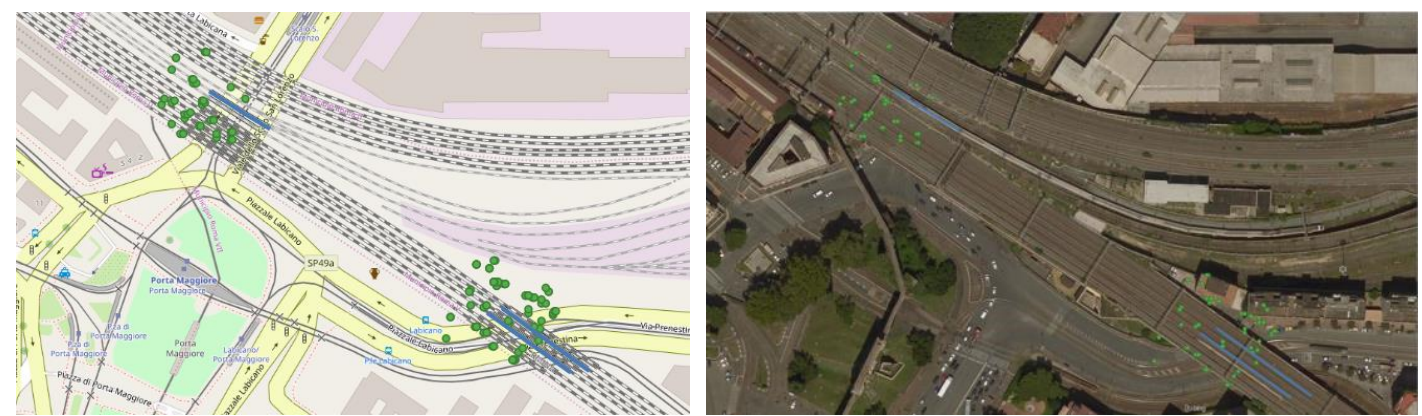

Figure 7. Outcomes of the application of the methodology. 
As these figures confirm, the methodology proved to be effective in providing useful information concerning potential subsidence recorded in the area pertaining to the structures. Such an information might be of paramount information for network administrators, in prioritizing on-site inspection and, therefore, increase the efficiency of their maintenance programs.

The potential of the tool is well depicted by Fig. 7, where a specific bridge is found to be characterized by different downlifting PS, corresponding to the position of the bridge pillars and having a displacement velocity ranging between -8 and $-4 \mathrm{~mm} /$ year.
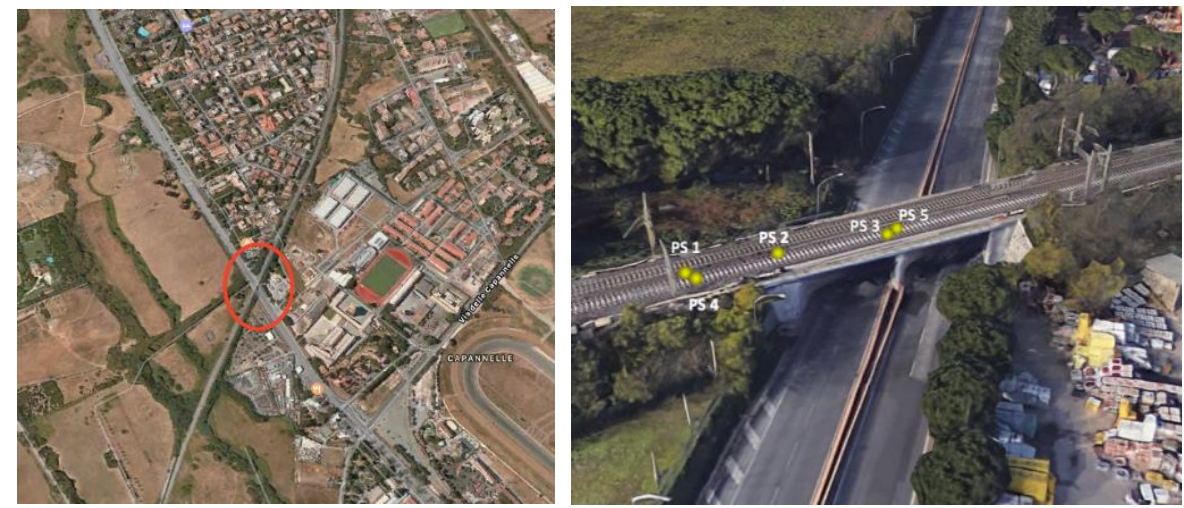

Figure 8. Outcomes of the application of the methodology on a specific bridge.

\section{CONCLUSIONS AND FUTURE DEVELOPMENTS}

This paper reports a methodology for the assessment of surface deformations of viaducts by reducing drastically computational time. To this purpose, a multi-stage automatic bridge monitoring protocol is developed at the network level by integration of information into Geographic Information System (GIS) catalogues and use of the InSAR imagery technique. The first stage locates viaducts in the area of interest by querying open data and inputting results into a GIS. On a second stage, an InSAR analysis of identified bridges is performed. This approach allows an estimate of surface displacements and an identification of bridge areas affected by millimeter settlements.

The methodology was finally tested on a specific study case concerning the monitoring of railway bridges and viaducts in Lazio Region, in central Italy, is reported as example of the suitability of the method for rapidly assess the occurrence of subsidence or differential settlements in the structures.

The method proved to be worthy of implementation in Planned Maintenance Systems (PMSs), as a viable solution for prioritizing the on-site monitoring efforts, thereby raising the effectiveness of the funds allocated on maintenance.

Among the future developments, algorithms capable of selecting the relevant areas where to run the SAR images processing are supposed to be developed, for increasing the productivity of the whole method.

*luca.bianchiniciampoli@uniroma3.it; phone +39065733 3617

\section{REFERENCES}

[1] Pines, D. and Aktan, A.E., "Status of structural health monitoring of long-span bridges in the United States", Prog. Struct. Mater. Eng., 4(4), 372-380 (2002).

[2] Ko, J.M. and Ni, Y.Q., "Technology developments in structural health monitoring of large-scale bridges", Eng. Struct., 27, 1715-1725 (2005).

[3] Alani, A.M., Aboutalebi, M., Kilic, G., "Applications of ground penetrating radar (GPR) in bridge deck monitoring and assessment”, J. Appl. Geo., 97, 45-54 (2013). 
[4] () OpenStreetMap contributors - https://www.openstreetmap.org/copyright

[5] Overpass API - http://overpass-api.de/

[6] Creative Commons Attribution-ShareAlike 3.0 licence (CC BY-SA)

[7] Ferretti, A., Prati, C. and Rocca, F., "Nonlinear subsidence rate estimation using permanent scatterers in differential SAR interferometry", IEEE Transactions on Geoscience and Remote Sensing, 38(5), 2202-2212 (2000)

[8] Ferretti, A., Prati, C. and Rocca, F., "Analysis of Permanent Scatterers in \{SAR \} Interferometry", (132), 1-3 (2000)

[9] Ferretti, A., Prati, C. and Rocca, F., "Permanent Scatters in SAR Interferometry", IEEE Transactions on Geoscience and Remote Sensing, 39(1), pp. 8-20 (2001)

[10]Colesanti, C., Le Mouelic, S., Bennani, M., Raucoules, D., Carnec, C. and Ferretti, A., "Detection of mining related ground instabilities using the permanent scatterers technique - A case study in the east of France", International Journal of Remote Sensing, 26(1), 201-207 (2005)

[11] Progetto Piano Straordinario di Telerilevamento - http://www.pcn.minambiente.it/mattm/progetto-pianostraordinario-di-telerilevamento/ 